\title{
Titanium oxo/alkoxo clusters with both phosphonate and methacrylate ligands
}

\author{
Matthias Czakler • Christine Artner • \\ Ulrich Schubert
}

Received: 6 October 2014/Accepted: 19 February 2015/Published online: 8 April 2015

(C) The Author(s) 2015. This article is published with open access at Springerlink.com

\begin{abstract}
The clusters $\mathrm{Ti}_{5} \mathrm{O}(\mathrm{O} i \mathrm{Pr})_{11}(\mathrm{OMc})\left(\mathrm{O}_{3} \mathrm{PR}\right)_{3}$ ( $\mathrm{OMc}=$ methacrylate $; \mathrm{R}=\mathrm{Et}, \quad \mathrm{CH}_{2} \mathrm{CH}_{2} \mathrm{CH}_{2} \mathrm{Br}$ ) and $\mathrm{Ti}_{10}(\mathrm{O} i \mathrm{Pr})_{16}(\mathrm{OMc})_{4}\left(\mathrm{O}_{3} \mathrm{PCH}_{2} \mathrm{CH}=\mathrm{CH}_{2}\right)_{10}$ were obtained when $\mathrm{Ti}(\mathrm{O} i \mathrm{Pr})_{4}$ was reacted with the corresponding bis(trimethylsilyl) phosphonate and methacrylic acid. Oxo clusters of the composition $\mathrm{Ti}_{6} \mathrm{O}_{4}(\mathrm{O} i \mathrm{Pr})_{10}(\mathrm{OMc})_{2}\left(\mathrm{O}_{3} \mathrm{PR}\right)_{2}$, with a variety of groups $\mathrm{R}\left(\mathrm{Et}, \mathrm{Ph}, \mathrm{CH}=\mathrm{CH}_{2}, \mathrm{CH}_{2} \mathrm{Ph}\right.$, $\mathrm{CH}_{2} \mathrm{CH}=\mathrm{CH}_{2}, \mathrm{CH}_{2} \mathrm{CH}_{2} \mathrm{CH}_{2} \mathrm{Br}, \mathrm{CH}_{2} \mathrm{CH}_{2} \mathrm{CN}, \mathrm{CH}_{2} \mathrm{C}(\mathrm{O}) \mathrm{Me}$, $\left.\mathrm{CH}_{2} \mathrm{CH}_{2} \mathrm{OC}(\mathrm{O}) \mathrm{C}(\mathrm{Me})=\mathrm{CH}_{2}\right)$, were formed instead, when a stoichiometric amount of water was added to the reaction mixture.

Graphical abstract
\end{abstract}

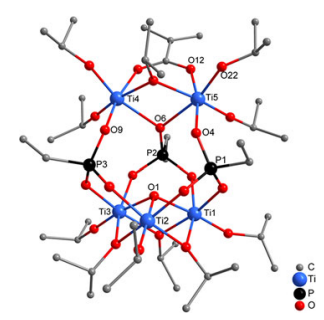

Keywords Titanium alkoxides - Methacrylate ligands . Phosphonate ligands $\cdot$ Structure analysis

\section{Introduction}

We have recently obtained phosphonate/acetate-substituted titanium oxo/alkoxo clusters from $\mathrm{Ti}(\mathrm{O} i \mathrm{Pr})_{4}$ and

M. Czakler · C. Artner · U. Schubert $(\bowtie)$

Institute of Materials Chemistry, Vienna University of

Technology, Vienna, Austria

e-mail: uschuber@mail.zserv.tuwien.ac.at bis(trimethylsilyl) phosphonates in the presence of acetic acid $(\mathrm{AcOH})$, which served for in situ water generation through ester formation with eliminated $i \mathrm{PrOH}$. Oxo clusters of the composition $\mathrm{Ti}_{6} \mathrm{O}_{4}(\mathrm{O} i \mathrm{Pr})_{10}(\mathrm{OAc})_{2}\left(\mathrm{O}_{3} \mathrm{PR}\right)_{2}$ were obtained with a large variety of functional and non-functional substituents $\mathrm{R}$ (Et, $\mathrm{CH}_{2} \mathrm{Ph}, \mathrm{CH}_{2} \mathrm{C}_{10} \mathrm{H}_{7}, \mathrm{CH}=\mathrm{CH}_{2}$, $\mathrm{CH}_{2} \mathrm{CH}=\mathrm{CH}_{2}, \mathrm{CH}_{2} \mathrm{CH}_{2} \mathrm{CH}_{2} \mathrm{Cl}, \mathrm{CH}_{2} \mathrm{CH}_{2} \mathrm{CH}_{2} \mathrm{Br}$ ), and also when the reaction conditions were varied [1]. This cluster type, which is also retained in solution, therefore appears to be very robust. Other clusters were only obtained in two exceptional cases (see below).

We extended these investigations by using methacrylic acid $(\mathrm{McOH})$ instead of acetic acid. Methacrylic acid could also produce water through in situ ester formation, but would additionally provide reactive ligands in the obtained clusters and thus allow incorporating such clusters in organic polymers by polymerization with organic comonomers (see review articles on cluster-crosslinked polymers [2, 3]). Especially the combination of ligands with different organic functionalities in one cluster appeared attractive. In this article, we report the outcome of these reactions.

\section{Results and discussion}

The cluster $\mathrm{Ti}_{5}\left(\mu_{3}-\mathrm{O}\right)\left(\mu_{2}-\mathrm{O} i \mathrm{Pr}\right)_{4}(\mathrm{O} i \mathrm{Pr})_{7}(\mathrm{OMc})\left(\mathrm{O}_{3} \mathrm{PEt}\right)_{3}(\mathbf{1})$ was formed when bis(trimethylsilyl) ethylphosphonate was reacted with methacrylic acid $(\mathrm{McOH})$ and $\mathrm{Ti}(\mathrm{O} i \mathrm{Pr})_{4}$ in a 1:1:3 molar ratio (Fig. 1). This cluster type was previously obtained, as an exception from general outcome of the reactions with acetic acid mentioned in the "Introduction", when bis(trimethylsilyl) 3-bromopropylphosphonate was reacted with acetic acid and $\mathrm{Ti}(\mathrm{O} i \mathrm{Pr})_{4}$ in a 1:1:2 ratio at room temperature. The asymmetric unit of crystalline $\mathbf{1}$ 


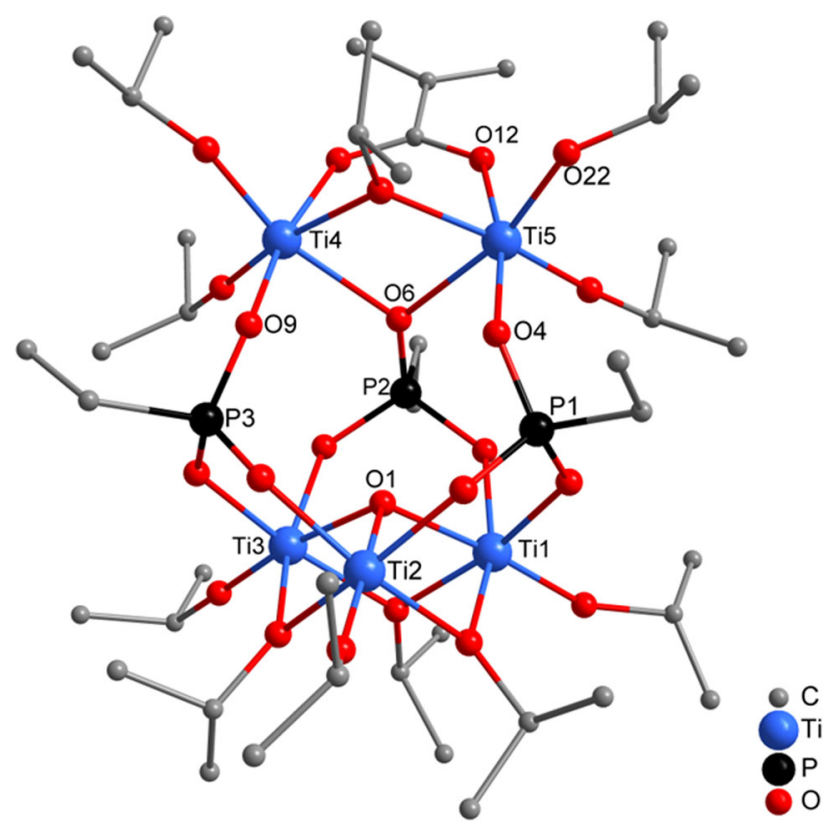

Fig. 1 Molecular structure of $\mathrm{Ti}_{5}\left(\mu_{3}-\mathrm{O}\right)\left(\mu_{2}-\mathrm{O} i \mathrm{Pr}\right)_{4}(\mathrm{O} i \mathrm{Pr})_{7}(\mathrm{OMc})$ $\left(\mathrm{O}_{3} \mathrm{PEt}\right)_{3}(\mathbf{1})$. Hydrogen atoms are omitted for clarity. Selected bond lengths/pm and angles $/^{\circ}: \operatorname{Ti}(1)-\mathrm{O}(1)$ 195.98(19), $\quad \mathrm{Ti}(1)-\mathrm{O}(3)$ 196.97(19), Ti(1)-O(13) 202.08(19), Ti(1)-O(17) 178.0(2), Ti(2)$\mathrm{O}(1)$ 194.0(2), $\mathrm{Ti}(2)-\mathrm{O}(2)$ 195.5(2), Ti(2)-O(13) 204.4(2), Ti(3)$\mathrm{O}(1)$ 196.5(2), $\mathrm{Ti}(3)-\mathrm{O}(19)$ 176.8(2), Ti(4)-O(6) 218.4(2), Ti(4)$\mathrm{O}(9)$ 195.4(2), $\mathrm{Ti}(5)-\mathrm{O}(4)$ 195.3(2), $\mathrm{Ti}(5)-\mathrm{O}(6)$ 221.2(2), Ti(5)$\mathrm{O}(12)$ 208.8(2), Ti(5)-O(22) 179.8(2), $\mathrm{P}(1)-\mathrm{O}(2)$ 153.8(2), $\mathrm{P}(1)-\mathrm{O}(4)$ 151.5(2), $\mathrm{P}(2)-\mathrm{O}(6)$ 153.9(2), $\mathrm{P}(3)-\mathrm{O}(9)$ 151.8(2); Ti(2)-O(1)-Ti(1) 105.18(8), Ti(4)-O(6)-Ti(5) 98.03(7)

contains two independent molecules with very similar bond distances and angles.

The structure of $\mathbf{1}$ is related to that of the clusters $\mathrm{Ti}_{4}\left(\mu_{3}-\mathrm{O}\right)\left(\mu_{2}-\mathrm{O} i \mathrm{Pr}\right)_{3}(\mathrm{O} i \mathrm{Pr})_{5}\left(\mathrm{O}_{3} \mathrm{PR}\right)_{3} \mathrm{~L}(\mathrm{~L}=$ neutral ligand $)$ [4-7], which consist of a symmetrical $\mathrm{Ti}_{3}\left(\mu_{3}-\mathrm{O}\right)\left(\mu_{2^{-}}\right.$ $\mathrm{O} i \mathrm{Pr})_{3}(\mathrm{O} i \mathrm{Pr})_{3}$ unit $(\mathrm{Ti}(1)-\mathrm{Ti}(3)$ in Fig. 1 to which a $\operatorname{Ti}(\mathrm{O} i \mathrm{Pr})_{2} \mathrm{~L}$ group is connected by means of three phosphonate ligands. In $\mathbf{1}$, the capping $\mathrm{Ti}(\mathrm{O} i \mathrm{Pr})_{2} \mathrm{~L}$ group is replaced by a $\mathrm{Ti}_{2}\left(\mu_{2}-\mathrm{O} i \mathrm{Pr}\right)(\mathrm{O} i \mathrm{Pr})_{4}\left(\mu_{2}-\mathrm{OMc}\right)$ moiety $(\mathrm{Ti}(4)$ and $\operatorname{Ti}(5)$ in Fig. 1). Two of the phosphonate ligands are coordinated to only one $\mathrm{Ti}$ atom of the $\mathrm{Ti}_{2}$ unit and have a 3.111 binding mode (w.xyz refers to the number of metal atoms to which the phosphonate ligand is coordinated [w], and the number of metal atoms to which each oxygen is coordinated $[x, y, z][8]$ ), while the third bridges both of them and has a binding mode of 4.211. The degree of condensation of $\mathbf{1}$ is $0.2(\mathrm{O} / \mathrm{Ti}$ ratio of the cluster core $)$, while it is 0.67 for the clusters $\mathrm{Ti}_{6} \mathrm{O}_{4}(\mathrm{O} i \mathrm{Pr})_{10^{-}}$ $(\mathrm{OAc})_{2}\left(\mathrm{O}_{3} \mathrm{PR}\right)_{2}$ obtained with acetic acid under the same conditions. This indicates that ester + water formation of methacrylic acid, relative to the rate of substitution [9], is slower than that of acetic acid.

${ }^{1} \mathrm{H},{ }^{13} \mathrm{C}$, and ${ }^{31} \mathrm{P}$ NMR spectra of re-dissolved crystals of 1 in $\mathrm{C}_{6} \mathrm{D}_{6}$ showed numerous signals. In the ${ }^{31} \mathrm{P}$ NMR spectrum, for example, eight resonances were observed, while two signals are expected if the solid-state structure of $\mathbf{1}$ was retained in solution. We therefore assume that $\mathbf{1}$ is in equilibrium with other compounds.

Isostructural $\quad \mathrm{Ti}_{5}\left(\mu_{3}-\mathrm{O}\right)\left(\mu_{2}-\mathrm{O} i \mathrm{Pr}\right)_{4}(\mathrm{O} i \mathrm{Pr})_{7}(\mathrm{OMc})-$ $\left(\mathrm{O}_{3} \mathrm{PCH}_{2} \mathrm{CH}_{2} \mathrm{CH}_{2} \mathrm{Br}\right)_{3}$ (2) was obtained from the reaction of bis(trimethylsilyl) bromopropylphosphonate, methacrylic acid, and $\mathrm{Ti}(\mathrm{O} i \mathrm{Pr})_{4}$ in a ratio of $1: 2: 3$. The higher proportion of $\mathrm{McOH}$ thus did not influence the outcome of the reaction. $\mathrm{Ti}_{2}(\mathrm{OMc})_{2}(\mathrm{O} i \mathrm{Pr})_{6} i \mathrm{PrOH}$ [8] was formed as a by-product, as proven by single crystal XRD. The ${ }^{1} \mathrm{H}$ and ${ }^{31} \mathrm{P}$ NMR spectra of the solid residue correspondingly showed numerous signals. Therefore, it can be assumed that a mixture of products was obtained and/or several species are in equilibrium with each other.

When a 1:2:3 mixture of bis(trimethylsilyl) allylphosphonate, methacrylic acid, and $\mathrm{Ti}(\mathrm{O} i \mathrm{Pr})_{4}$ was heated to reflux, the complex $\mathrm{Ti}_{10}\left(\mu_{2}-\mathrm{O} i \mathrm{Pr}\right)_{2}(\mathrm{O} i \mathrm{Pr})_{14}(\mathrm{OMc})_{4}$ $\left(\mathrm{O}_{3} \mathrm{PCH}_{2} \mathrm{CH}=\mathrm{CH}_{2}\right)_{10}$ (3) (Fig. 2) was obtained after crystallization from $\mathrm{CH}_{2} \mathrm{Cl}_{2}$. It is noteworthy that 3 contains no oxo groups, but more $\mathrm{O} i \mathrm{Pr}$ groups were substituted by $\mathrm{OMc}$ or $\mathrm{O}_{3} \mathrm{PR}$ ligands compared to $\mathbf{1}$ and $\mathbf{2}$. The different outcome of this reaction, compared to $\mathbf{1}$ and $\mathbf{2}$, may be due to the higher reaction temperature. We have previously shown that higher reaction temperatures favor substitution over ester formation [9].

The structure of 3 consists of two $\mathrm{Ti}_{5}(\mathrm{O} i \mathrm{Pr})_{8}(\mathrm{OMc})_{2}$ $\left(\mathrm{O}_{3} \mathrm{P} \text {-allyl }\right)_{5}$ units, which are bridged by two (3.111) phosphonate ligands. The $\mathrm{Ti}_{5}$ units are composed of methacrylate-bridged dimers $\mathrm{Ti}_{2}\left(\mu_{2}-\mathrm{O} i \mathrm{Pr}\right)(\mathrm{OiPr})_{3}(\mathrm{OMc})$ $(\mathrm{Ti}(2), \mathrm{Ti}(5))$ and $\mathrm{Ti}_{2}(\mathrm{Oi} \mathrm{Pr})_{3}(\mathrm{OMc})(\mathrm{Ti}(3), \mathrm{Ti}(4))$, respectively, which are connected through phosphonate ligands among each other as well as to the fifth titanium atom (Ti(1)). Each of the octahedrally coordinated titanium atoms is at least bound to two different phosphonate ligands; $\operatorname{Ti}(1)$ is coordinated by five different oxygen atoms of phosphonate ligands and one OiPr ligand. The complexity of the structure of $\mathbf{3}$ is also reflected in the different binding modes of the phosphonate ligands, of which six are 3.111, two are 3.211, and two are 4.211.

The reactions leading to $\mathbf{1}, \mathbf{2}$, and $\mathbf{3}$ show that clusters with a noticeably lower degree of condensation were formed compared to analogous reactions with acetic acid [1]. This is most probably due to the lower reaction rate of ester formation between methacrylic acid and isopropyl alcohol compared to that of acetic acid [10]. This assumption was proven by the deliberate addition of water to the reaction mixture. Thus, when bis(trimethylsilyl) 3-bromopropylphosphonate, methacrylic acid, $\mathrm{Ti}(\mathrm{O} i \mathrm{Pr})_{4}$, and water were reacted in a 1:1:3:2 ratio, the cluster $\mathrm{Ti}_{6} \mathrm{O}_{4}(\mathrm{O} i \mathrm{Pr})_{10}$ $(\mathrm{OMc})_{2}\left(\mathrm{O}_{3} \mathrm{PCH}_{2} \mathrm{CH}_{2} \mathrm{CH}_{2} \mathrm{Br}\right)_{2}$ (4) (Fig. 3) was obtained. The cluster 4 is isostructural to $\mathrm{Ti}_{6} \mathrm{O}_{4}(\mathrm{OiPr})_{10}(\mathrm{OAc})_{2}$ $\left(\mathrm{O}_{3} \mathrm{PCH}_{2} \mathrm{CH}_{2} \mathrm{CH}_{2} \mathrm{Br}\right)_{2}$ obtained with acetic acid [1]. 


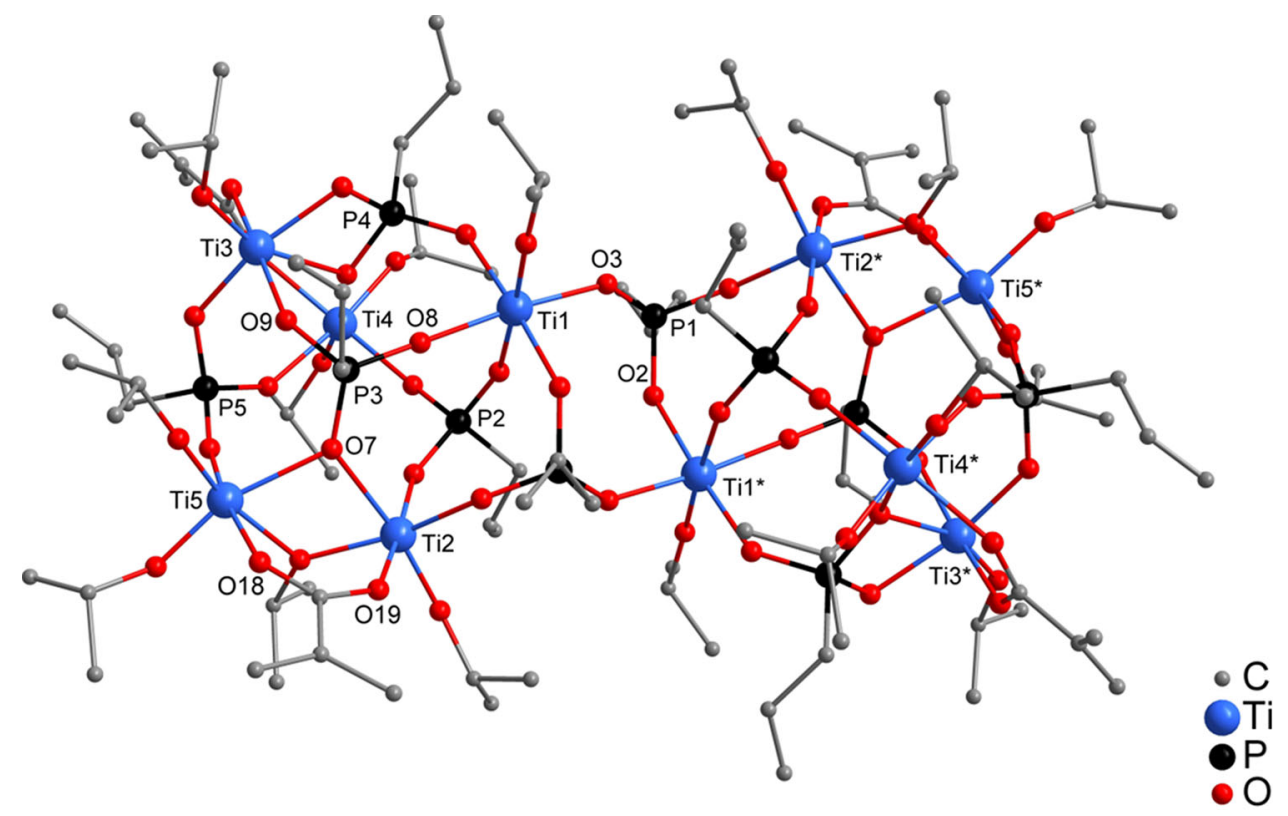

Fig. 2 Molecular structure of $\mathrm{Ti}_{10}\left(\mu_{2}-\mathrm{O} i \mathrm{Pr}\right)_{2}(\mathrm{O} i \mathrm{Pr})_{14}(\mathrm{OMc})_{4}$ $\left(\mathrm{O}_{3} \mathrm{PCH}_{2} \mathrm{CH}=\mathrm{CH}_{2}\right)_{10}$ (3). Hydrogen atoms are omitted for clarity. Selected bond lengths/pm and angles/ $/{ }^{\circ}: \operatorname{Ti}(1)-\mathrm{O}(2)$ 193.6(2), $\mathrm{Ti}(1)-\mathrm{O}(3)$ 195.9(2), $\mathrm{Ti}(1)-\mathrm{O}(4) \quad 202.1(2), \quad \mathrm{Ti}(1)-\mathrm{O}(8)$ 195.8(2), $\mathrm{Ti}(1)-\mathrm{O}(12) \quad 200.9(2), \mathrm{Ti}(1)-\mathrm{O}(20)$ 175.6(2), Ti(2)-O(7) 216.5(2), $\mathrm{Ti}(2)-\mathrm{O}(19)$ 206.3(3), Ti(3)-O(9) 193.5(2), Ti(3)-O(10) 221.1(2),

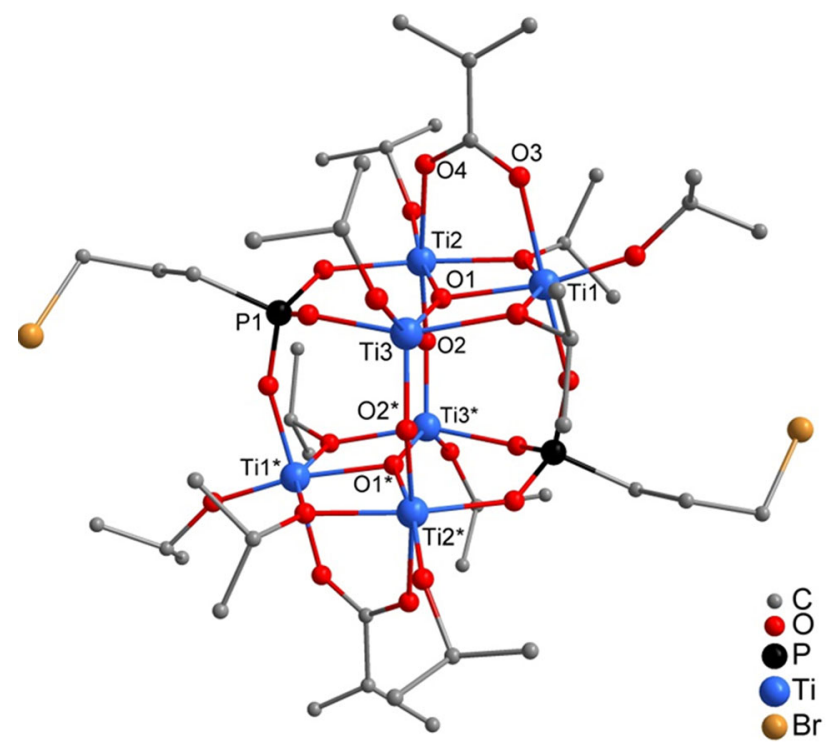

Fig. 3 Molecular structure of $\mathrm{Ti}_{6} \mathrm{O}_{4}(\mathrm{O} i \mathrm{Pr})_{10}(\mathrm{OMc})_{2^{-}}$ $\left(\mathrm{O}_{3} \mathrm{PCH}_{2} \mathrm{CH}_{2} \mathrm{CH}_{2} \mathrm{Br}\right)_{2}$ (4). Hydrogen atoms are omitted for clarity. Selected bond lengths/pm and angles ${ }^{\circ}$ : $\mathrm{Ti}(1)-\mathrm{O}(1)$ 197.1(1), $\mathrm{Ti}(1)-$ O(3) 208.8(1), Ti(1)-O(5) 199.0(1), Ti(1)-O(6) 196.1(1), Ti(1)-O(7) 177.7(1), $\mathrm{Ti}(1)-\mathrm{O}(12) \quad 192.9(1), \quad \mathrm{Ti}(2)-\mathrm{O}(1)$ 199.2(1), $\mathrm{Ti}(2)-\mathrm{O}(2)$ 187.3(1), $\mathrm{Ti}(2)-\mathrm{O}(10)$ 196.5(1), $\mathrm{Ti}(3)-\mathrm{O}(1)$ 189.9(1), $\mathrm{Ti}(3)-\mathrm{O}(2) *$ 175.0(1), Ti(3)-O(6) 204.7(1), Ti(3)-O(9) 180.9(1); Ti(3)-O(1)-Ti(1) 105.83(6), Ti(3)-O(1)-Ti(2) 149.36(7), Ti(1)-O(1)-Ti(2) 104.15(6), $\mathrm{Ti}(3)-\mathrm{O}(2)-\mathrm{Ti}(2) \quad 148.49(8), \quad \mathrm{Ti}(1)-\mathrm{O}(5)-\mathrm{Ti}(2) \quad 102.48(6), \quad \mathrm{Ti}(1)-$ $\mathrm{O}(6)-\mathrm{Ti}(3) 100.75(5)$
$\mathrm{Ti}(4)-\mathrm{O}(5)$ 193.1(2), Ti(5)-O(7) 217.4(2), Ti(5)-O(18) 206.8(3); $\mathrm{Ti}(2)-\mathrm{O}(7)-\mathrm{Ti}(5) \quad 99.38(9), \quad \mathrm{Ti}(2)-\mathrm{O}(21)-\mathrm{Ti}(5) \quad 110.3(1), \quad \mathrm{Ti}(3)-$ $\mathrm{O}(10)-\mathrm{Ti}(4)$ 126.8(1), O(1)-P(1)-O(2) 110.7(1), O(1)-P(1)-C(1A) 107.3(2), O(10)-P(4)-O(11) 99.1(1), O(10)-P(4)-O(12) 114.6(1), $\mathrm{O}(11)-\mathrm{P}(4)-\mathrm{O}(12) 115.6(1)$

The centrosymmetric cluster $\mathbf{4}$ is isostructural to the previously reported acetate-substituted clusters $\mathrm{Ti}_{6} \mathrm{O}_{4}(\mathrm{O} i$ $\mathrm{Pr})_{10}(\mathrm{OAc})_{2}\left(\mathrm{O}_{3} \mathrm{PR}\right)_{2}$ [1]. The cluster core is formed by two parallel, unsymmetrically substituted $\mathrm{Ti}_{3}\left(\mu_{3}-\mathrm{O}\right)\left(\mu_{2}-\right.$ $\mathrm{O} i \mathrm{Pr})_{2}(\mathrm{O} i \mathrm{Pr})_{3}\left(\mu_{2}-\mathrm{OMc}\right)$ units connected by $\mu_{2}$-oxo $(\mathrm{O}(2)$ and $\mathrm{O}(2)^{*}, *$ denotes symmetry-related atoms) and phosphonate bridges. $\operatorname{Ti}(1)$ and $\operatorname{Ti}(2)$ are bridged by both an $\mathrm{O} i \mathrm{Pr}$ and a methacrylate ligand and are octahedrally coordinated while $\mathrm{Ti}(3)$ has a distorted trigonal bipyramidal coordination sphere. The central $\mathrm{Ti}_{3} \mathrm{O}$ unit is unsymmetrical, with one short $(\mathrm{Ti}(3)-\mathrm{O}(1)$ 189.9(1) pm) and two long $\mathrm{Ti}-\mathrm{O}$ distances $(\mathrm{Ti}(1)-\mathrm{O}(1)$ 197.1(1), Ti(2)-O(1) 199.2(2) pm), as in the acetate derivatives $\mathrm{Ti}_{6} \mathrm{O}_{4}(\mathrm{O} i \mathrm{Pr})_{10^{-}}$ $(\mathrm{OAc})_{2}\left(\mathrm{O}_{3} \mathrm{PR}\right)_{2}$.

NMR data show that the structure of $\mathbf{4}$, especially also their inversion symmetry is retained in solution. Thus, one signal was observed in the ${ }^{31} \mathrm{P}$ NMR spectrum at $27.34 \mathrm{ppm}$. In the ${ }^{1} \mathrm{H}$ NMR spectrum five doublets for the methyl groups of the $\mathrm{O} i \mathrm{Pr}$ ligands were observed and three signals for the $\mathrm{CH}$ groups (at 4.86, 4.97, and $5.33 \mathrm{ppm}$ ) the latter two with double intensity. One singlet at $2.08 \mathrm{ppm}$ and two multiplets at 5.41 and $6.36 \mathrm{ppm}$ can be assigned to the two OMc ligands. In the ${ }^{13} \mathrm{C}$ NMR spectrum only one doublet for each $\mathrm{P}_{-} \mathrm{CH}_{2}$ group was found and one set of signals for the OMc ligands. The signals of the $\mathrm{O} i \mathrm{Pr}$ ligands were partly overlapping. 
The clusters 5-12 with a great variety of functional or non-functional phosphonate ligands were obtained according to Scheme 1 by the same synthesis procedure as that for 4. The ${ }^{1} \mathrm{H},{ }^{31} \mathrm{P}$, and ${ }^{13} \mathrm{C}$ NMR spectra of 5-12 are similar to that of 4 .

\section{Conclusions}

The first step in reactions of metal alkoxides with carboxylic or phosphonic acids is the substitution of an OR ligand by a carboxylate or phosphonate ligand. The thus liberated alcohol can undergo ester formation with the carboxylic or phosphonic acid, which produces water that hydrolyzes part or all of the remaining M-OR groups. Thus two reactions, viz. substitution and ester formation, compete with each other, and their relative rate is one of the decisive parameters influencing the outcome of such reactions. How the clusters are formed from the initially formed $\mathrm{M}(\mathrm{OR})_{x}$ (carboxylate/phosphonate $)_{y}$ derivatives has not been elucidated in any case. The situation becomes even more complex when two different metal alkoxides or, as in the present case, two different acids are involved.

In previous work, we had preferentially obtained phosphonate/acetate-substituted titanium oxo/alkoxo clusters of the composition $\mathrm{Ti}_{6} \mathrm{O}_{4}(\mathrm{O} i \mathrm{Pr})_{10}(\mathrm{OAc})_{2}\left(\mathrm{O}_{3} \mathrm{PR}\right)_{2}$ from $\mathrm{Ti}(\mathrm{OiPr})_{4}$ and bis(trimethylsilyl) phosphonates in the presence of acetic acid (AcOH) [1]. The results of the work reported in this article show that the degree of condensation of the obtained clusters ( $\mathbf{1}$ and $\mathbf{2}$ ) was lower when acetic acid was replaced by methacrylic acid. In one case, the product (compound 3) contained no oxo groups at all. This can be taken as evidence that the rate of esterification of methacrylic acid is lower than that of acetic acid.

The lower esterification rate can be compensated, however, by controlled addition of a stoichiometric amount of "external" water. The thus obtained methacrylate/ phosphonate-substituted clusters 4-12, with a very wide variety of phosphonate ligands, are isostructural to the acetate-substituted clusters $\mathrm{Ti}_{6} \mathrm{O}_{4}(\mathrm{O} i \mathrm{Pr})_{10}(\mathrm{OAc})_{2}\left(\mathrm{O}_{3} \mathrm{PR}\right)_{2}$ obtained in earlier experiments [1]. Incorporation of the polymerizable OMc ligands is a very interesting option for the preparation of cluster-crosslinked polymers [2, 3], especially because this allows the combination (a) of reactive and non-reactive ligands as well as (b) ligands with different organic functionalities in a controlled manner in one cluster.

\section{Experimental}

All operations were carried out in a moisture- and oxygenfree argon atmosphere using Schlenk techniques. Isopropyl alcohol was dried by refluxing twice over sodium metal and distillation. The bis(trimethylsilyl) phosphonates were prepared as reported before [1].

\section{Methacrylate-phoshonate-substituted $T_{i}$ oxo clusters}

$\mathrm{Ti}_{5} \mathrm{O}(\mathrm{OiPr})_{11}(\mathrm{OMc})\left(\mathrm{O}_{3} \mathrm{PEt}\right)_{3} \quad(\mathbf{1}): 1.6 \mathrm{~cm}^{3}$ of $\mathrm{Ti}(\mathrm{O} i \mathrm{Pr})_{4}$ $(5.42 \mathrm{mmol})$ was added to a solution of $500 \mathrm{~mm}^{3}$ of bis(trimethylsilyl) ethylphosphonate $(1.81 \mathrm{mmol})$ and $153 \mathrm{~mm}^{3}$ of methacrylic acid $(1.81 \mathrm{mmol})$ in $2 \mathrm{~cm}^{3}$ of isopropyl alcohol. Crystals of $\mathbf{1}$ were obtained from this solution after 8 weeks. Yield $410 \mathrm{mg}(35 \%)$.

$\mathrm{Ti}_{5} \mathrm{O}(\mathrm{OiPr})_{11}(\mathrm{OMc})\left(\mathrm{O}_{3} \mathrm{PCH}_{2} \mathrm{CH}_{2} \mathrm{CH}_{2} \mathrm{Br}\right)_{3}(\mathbf{2}): 1.2 \mathrm{~cm}^{3}$ of $\mathrm{Ti}(\mathrm{O} i \mathrm{Pr})_{4}(4.1 \mathrm{mmol})$ was added to a solution of $400 \mathrm{~mm}^{3}$ of bis(trimethylsilyl) 3-bromopropylphosphonate (1.34 mmol) in $2 \mathrm{~cm}^{3}$ of $i \mathrm{PrOH}$, followed by addition of $113 \mathrm{~mm}^{3}$ of methacrylic acid $(1.34 \mathrm{mmol})$. Crystals of 2 were obtained from this solution after 3 weeks. Yield $620 \mathrm{mg}$ (mixture of compounds).
Scheme 1

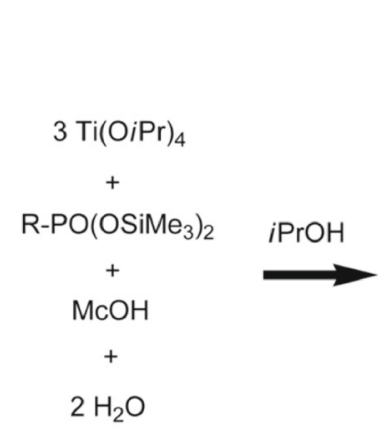

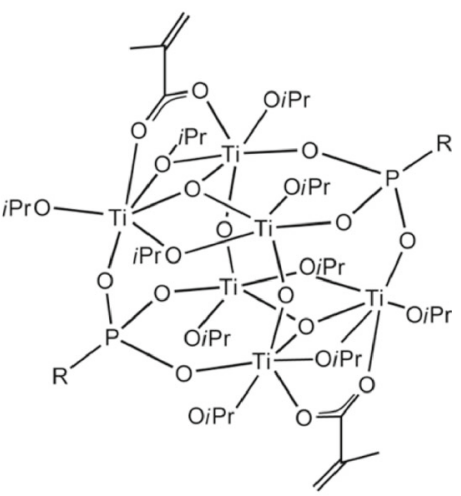

R

$4 \mathrm{CH}_{2} \mathrm{CH}_{2} \mathrm{CH}_{2} \mathrm{Br}$

$5 \mathrm{Ph}$

$6 \mathrm{CH}=\mathrm{CH}_{2}$

$7 \mathrm{CH}_{2} \mathrm{Ph}$

$8 \mathrm{CH}_{2} \mathrm{CH}=\mathrm{CH}_{2}$

$9 \mathrm{CH}_{2} \mathrm{CH}_{2} \mathrm{OC}(\mathrm{O}) \mathrm{C}(\mathrm{Me})=\mathrm{CH}_{2}$

$10 \mathrm{CH}_{2} \mathrm{CH}_{2} \mathrm{CN}$

$11 \mathrm{Et}$

$12 \mathrm{CH}_{2} \mathrm{C}(\mathrm{O}) \mathrm{Me}$ 
Methacrylate-phoshonate-substituted Ti $i_{10}$ oxo cluster $\mathrm{Ti}_{10}(\mathrm{OiPr})_{16}(\mathrm{OMc})_{4}\left(\mathrm{O}_{3} \mathrm{PCH}_{2} \mathrm{CHCH}_{2}\right)_{10}$ (3):

$7.1 \mathrm{~cm}^{3}$ of $\mathrm{Ti}(\mathrm{O} i \mathrm{Pr})_{4}(24 \mathrm{mmol})$ was added to a solution of $2 \mathrm{~cm}^{3}$ of bis(trimethylsilyl) allylphosphonate $(8 \mathrm{mmol})$ and $1.35 \mathrm{~cm}^{3}$ of methacrylic acid $(16 \mathrm{mmol})$ in $12 \mathrm{~cm}^{3}$ of isopropyl alcohol. The solution was heated to reflux for $16 \mathrm{~h}$, and a suspension was formed. The solid was separated by filtration and recrystallized from $\mathrm{CH}_{2} \mathrm{Cl}_{2}$. Yield $120 \mathrm{mg} \mathrm{(5 \% ).} \mathrm{The} \mathrm{crystals} \mathrm{could} \mathrm{not} \mathrm{be} \mathrm{re-dissolved}$ in $\mathrm{CD}_{2} \mathrm{Cl}_{2}$ or another non-coordinating organic solvent and therefore no NMR measurements were performed.

Methacrylate-phoshonate-substituted $\mathrm{Ti}_{6}$ oxo clusters: $\mathrm{Ti}_{6} \mathrm{O}_{4}(\mathrm{OiPr})_{10}(\mathrm{OMc})_{2}\left(\mathrm{O}_{3} \mathrm{PCH}_{2} \mathrm{CH}_{2} \mathrm{CH}_{2} \mathrm{Br}\right)_{2}$ (4) and $\mathrm{Ti}_{6} \mathrm{O}_{4}(\mathrm{OiPr})_{10}(\mathrm{OMc})_{2}\left(\mathrm{O}_{3} \mathrm{PPh}\right)_{2}(\mathbf{5})$

$\mathrm{Ti}(\mathrm{OiPr})_{4}\left(1.17 \mathrm{~cm}^{3}, 4 \mathrm{mmol}\right)$ was quickly added to a solution of $400 \mathrm{~mm}^{3}$ of bis(trimethylsilyl) 3-bromopropylphosphonate $(1.3 \mathrm{mmol}$ ) [or $225 \mathrm{mg}$ of bis(trimethylsilyl) phenylphosphonate $(0.82 \mathrm{mmol})]$ in $2 \mathrm{~cm}^{3}$ of 2-propanol followed by addition of $110 \mathrm{~mm}^{3}$ of methacrylic acid $(1.3 \mathrm{mmol})$. Finally, $48 \mathrm{~mm}^{3}$ of water $(2.7 \mathrm{mmol})$ diluted in $1 \mathrm{~cm}^{3}$ of 2-propanol was injected quickly directly into the solution. Crystals were obtained after 1 week.

4: yield $420 \mathrm{mg}(43 \%) ;{ }^{1} \mathrm{H}$ NMR $\left(\mathrm{C}_{6} \mathrm{D}_{6}, 250 \mathrm{MHz}\right)$ : $\delta=1.31\left(\mathrm{~d},{ }^{3} J_{\mathrm{H}, \mathrm{H}}=6.09 \mathrm{~Hz}, 12 \mathrm{H}, \mathrm{CHMe}\right), 1.41(\mathrm{~d}$, $\left.{ }^{3} J_{\mathrm{H}, \mathrm{H}}=6.24 \mathrm{~Hz}, 12 \mathrm{H}, \mathrm{CHMe}\right), 1.48\left(\mathrm{~d},{ }^{3} J_{\mathrm{H}, \mathrm{H}}=6.09 \mathrm{~Hz}\right.$, $12 \mathrm{H}, \mathrm{CHMe}), 1.74\left(\mathrm{~d},{ }^{3} J_{\mathrm{H}, \mathrm{H}}=6.24 \mathrm{~Hz}, 12 \mathrm{H}, \mathrm{CHMe}\right), 1.82$ $\left(\mathrm{d},{ }^{3} J_{\mathrm{H}, \mathrm{H}}=6.24 \mathrm{~Hz}, 12 \mathrm{H}, \mathrm{CHMe}\right), 1.72-1.88(\mathrm{~m}, 2 \mathrm{H}$, $\left.\mathrm{PCH}_{2}\right), 2.08\left(\mathrm{~s}, 6 \mathrm{H},=\mathrm{CCH}_{3}\right), 2.36\left(\mathrm{~m},{ }^{3} J_{\mathrm{P}, \mathrm{H}}=15.84 \mathrm{~Hz}\right.$, $\left.{ }^{3} J_{\mathrm{H}, \mathrm{H}}=7.31 \mathrm{~Hz}, \quad 2 \mathrm{H}, \quad \mathrm{CH}_{2} \mathrm{CH}_{2} \mathrm{P}\right), \quad 3.46 \quad(\mathrm{t}$, $\left.{ }^{3} J_{\mathrm{H}, \mathrm{H}}=7.16 \mathrm{~Hz}, \mathrm{CH}_{2} \mathrm{Br}\right), 4.86\left(\mathrm{~m},{ }^{3} J_{\mathrm{H}, \mathrm{H}}=6.17 \mathrm{~Hz}, 2 \mathrm{H}\right.$, $\mathrm{OCH}), 4.97\left(\mathrm{~m},{ }^{3} J_{\mathrm{H}, \mathrm{H}}=6.13 \mathrm{~Hz}, 4 \mathrm{H}, \mathrm{OCH}\right), 5.33(\mathrm{~m}$, $\left.{ }^{3} J_{\mathrm{H}, \mathrm{H}}=6.20 \mathrm{~Hz}, 4 \mathrm{H}, \mathrm{OCH}\right), 5.39-5.43\left(\mathrm{~m}, 2 \mathrm{H},=\mathrm{CH}_{2}\right)$, 6.34-6.38 (m, 2H, $\left.=\mathrm{CH}_{2}\right)$ ppm; ${ }^{31} \mathrm{P}$ NMR $\left(\mathrm{C}_{6} \mathrm{D}_{6}\right.$, 101.2 MHz): $\delta=27.34 \mathrm{ppm} ; \quad{ }^{13} \mathrm{C} \quad \mathrm{NMR} \quad\left(\mathrm{C}_{6} \mathrm{D}_{6}\right.$, $62.9 \mathrm{MHz}): \delta=18.63$ (CHMe), 23.89 (CHMe), 24.20 (CHMe), 24.81 (CHMe), 25.20 (CHMe), 25.67 (d, $\left.{ }^{1} J_{\mathrm{P}, \mathrm{C}}=157 \mathrm{~Hz}, \quad \mathrm{PCH}_{2}\right), \quad 27.68 \quad\left(\mathrm{~d}, \quad{ }^{2} J_{\mathrm{P}, \mathrm{C}}=4.53 \mathrm{~Hz}\right.$, $\left.\mathrm{CH}_{2} \mathrm{CH}_{2} \mathrm{P}\right), 33.81\left(\mathrm{~d},{ }^{3} \mathrm{~J}_{\mathrm{P}, \mathrm{C}}=14.96 \mathrm{~Hz}, \mathrm{CH}_{2} \mathrm{Br}\right), 77.83$ $(\mathrm{OCH}), 78.69(\mathrm{OCH}), 79.40(\mathrm{OCH}), 123.41\left(=\mathrm{CH}_{2}\right)$, 140.02 (=CMe-), $173.39(\mathrm{COO}) \mathrm{ppm}$.

5: yield $160 \mathrm{mg}(27 \%) ;{ }^{1} \mathrm{H}$ NMR $\left(\mathrm{C}_{6} \mathrm{D}_{6}, 250 \mathrm{MHz}\right)$ : $\delta=1.26\left(\mathrm{~d},{ }^{3} J_{\mathrm{H}, \mathrm{H}}=6.13 \mathrm{~Hz}, 12 \mathrm{H}, \mathrm{CHMe}\right), 1.43(\mathrm{~d}$, $\left.{ }^{3} J_{\mathrm{H}, \mathrm{H}}=6.08 \mathrm{~Hz}, 24 \mathrm{H}, \mathrm{CH} M e\right), 1.82\left(\mathrm{~d},{ }^{3} J_{\mathrm{H}, \mathrm{H}}=6.40 \mathrm{~Hz}\right.$, $12 \mathrm{H}, \mathrm{CHMe}), 1.84\left(\mathrm{~d},{ }^{3} J_{\mathrm{H}, \mathrm{H}}=6.40 \mathrm{~Hz}, 12 \mathrm{H}, \mathrm{CHMe}\right), 2.14$ (s, 6H, $\left.\mathrm{CH}_{3}(\mathrm{OMc})\right), 4.88\left(\mathrm{~m},{ }^{3} \mathrm{~J}_{\mathrm{H}, \mathrm{H}}=6.17 \mathrm{~Hz}, 2 \mathrm{H}, \mathrm{CH}\right.$ $(\mathrm{O} i \mathrm{Pr})), 4.99\left(\mathrm{~m},{ }^{3} J_{\mathrm{H}, \mathrm{H}}=6.13 \mathrm{~Hz}, 4 \mathrm{H}, \mathrm{CH}(\mathrm{O} i \mathrm{Pr})\right)$, 5.36-5.58 (m, 6H, CH (OiPr) + $\left.\mathrm{CH}_{2}(\mathrm{OMc})\right), 6.43-6.46$ $\left(\mathrm{m}, 2 \mathrm{H}, \mathrm{CH}_{2}(\mathrm{OMc})\right), 7.10-7.28(\mathrm{~m}, 2 \mathrm{H}, \mathrm{CH}(\mathrm{Ph}))$, 7.34-7.44 (m, 4H, Ph), 8.33-8.44 (m, 4H, Ph) ppm; ${ }^{31} \mathrm{P}$ NMR $\left(\mathrm{C}_{6} \mathrm{D}_{6}, 101.2 \mathrm{MHz}\right): \delta=16.03 \mathrm{ppm} ;{ }^{13} \mathrm{C}$ NMR $\left(\mathrm{C}_{6} \mathrm{D}_{6}, 62.9 \mathrm{MHz}\right): \delta=18.64(\mathrm{CHMe}), 24.06(\mathrm{CHMe})$,
24.26 (CHMe), 24.83 (CHMe), 25.17 (CHMe), 77.93 $(\mathrm{OCH}), 78.78(\mathrm{OCH}), 79.48(\mathrm{OCH}), 123.28\left(=\mathrm{CH}_{2}\right)$, $130.25\left(\mathrm{~d}, J_{\mathrm{P}, \mathrm{C}}=2.99 \mathrm{~Hz}, \mathrm{Ph}\right), 130.99\left(\mathrm{~d}, J_{\mathrm{P}, \mathrm{C}}=9.36 \mathrm{~Hz}\right.$, $\mathrm{Ph}), \quad 131.80 \quad\left(\mathrm{~d}, \quad J_{\mathrm{P}, \mathrm{C}}=9.97 \mathrm{~Hz}, \quad \mathrm{Ph}\right), 134.50 \quad(\mathrm{~d}$, $\left.{ }^{1} J_{\mathrm{P}, \mathrm{C}}=208.43 \mathrm{~Hz}, \mathrm{Ph}\right), 140.25(=\mathrm{CMe}-), 173.46(\mathrm{COO})$ ppm.

$\mathrm{Ti}_{6} \mathrm{O}_{4}(\mathrm{OiPr})_{10}(\mathrm{OMc})_{2}\left(\mathrm{O}_{3} \mathrm{P}-\mathrm{CH}=\mathrm{CH}_{2}\right)_{2} \quad(\mathbf{6}), \quad \mathrm{Ti}_{6} \mathrm{O}_{4}(\mathrm{Oi}-$ $\left.\mathrm{Pr})_{10} \mathrm{OMC}\right)_{2}\left(\mathrm{O}_{3} \mathrm{PCH}_{2} \mathrm{Ph}\right)_{2} \quad$ (7), $\quad \mathrm{Ti}_{6} \mathrm{O}_{4}(\mathrm{OiPr})_{10}(\mathrm{OMc})_{2}-$ $\left(\mathrm{O}_{3} \mathrm{PCH}_{2}-\mathrm{CH}=\mathrm{CH}_{2}\right)_{2}(8), \mathrm{Ti}_{6} \mathrm{O}_{4}(\mathrm{OiPr})_{10}(\mathrm{OMc})_{2}\left(\mathrm{O}_{3} \mathrm{PCH}_{2}-\right.$ $\left.\mathrm{CH}_{2}-\mathrm{OMc}\right)_{2} \quad$ (9), $\quad \mathrm{Ti}_{6} \mathrm{O}_{4}(\mathrm{OiPr})_{10}(\mathrm{OMc})_{2}\left(\mathrm{O}_{3} \mathrm{PCH}_{2} \mathrm{CH}_{2^{-}}\right.$ $\mathrm{C} \equiv \mathrm{N}_{2} \quad(\mathbf{1 0}), \quad \mathrm{Ti}_{6} \mathrm{O}_{4}(\mathrm{OiPr})_{10}(\mathrm{OMc})_{2}\left(\mathrm{O}_{3} \mathrm{PCH}_{2} \mathrm{CH}_{3}\right)_{2} \quad$ (11), $\mathrm{Ti}_{6} \mathrm{O}_{4}(\mathrm{OiPr})_{10}(\mathrm{OMc})_{2}\left(\mathrm{O}_{3} \mathrm{PCH}_{2} \mathrm{COCH}_{3}\right)_{2}(\mathbf{1 2})$.

Compared to $\mathbf{4}$ and $\mathbf{5}$, the synthesis was slightly modified. In the synthesis of $6660 \mathrm{~mm}^{3}$ of $\mathrm{Ti}(\mathrm{O} i \mathrm{Pr})_{4}$ $(2.27 \mathrm{mmol})$ was added to a mixture of $200 \mathrm{~mm}^{3}$ of bis(trimethyl)silyl vinylphosphonate $(0.76 \mathrm{mmol})$ and $64 \mathrm{~mm}^{3}$ of methacrylic acid $(0.76 \mathrm{mmol})$ in $2 \mathrm{~cm}^{3}$ of 2-propanol. Immediately afterwards, $27.3 \mathrm{~mm}^{3}$ of water (1.52 mmol) diluted in $0.5 \mathrm{~cm}^{3}$ of 2-propanol was added. Crystals of 6 were obtained after 3 days. The syntheses of 7-11 were analogous. The synthesis of $\mathbf{1 2}$ was done analogously, but the precursor solution was additionally heated after addition of water until a clear solution was obtained.

6: yield $70 \mathrm{mg}(14 \%) ;{ }^{1} \mathrm{H}$ NMR $\left(\mathrm{C}_{6} \mathrm{D}_{6}, 250 \mathrm{MHz}\right)$ : $\delta=1.33\left(\mathrm{~d},{ }^{3} J_{\mathrm{H}, \mathrm{H}}=6.09 \mathrm{~Hz}, 12 \mathrm{H}\right.$, OCHMe $), 1.41(\mathrm{~d}$, ${ }^{3} J_{\mathrm{H}, \mathrm{H}}=6.09 \mathrm{~Hz}, \quad 12 \mathrm{H}, \quad$ OCHMe $), \quad 1.51 \quad\left(\mathrm{~d},{ }^{3} J_{\mathrm{H}, \mathrm{H}}=\right.$ $5.94 \mathrm{~Hz}, 12 \mathrm{H}$, OCHMe), $1.79\left(\mathrm{~d},{ }^{3} J_{\mathrm{H}, \mathrm{H}}=6.24 \mathrm{~Hz}, 12 \mathrm{H}\right.$, OCHMe $), 1.84\left(\mathrm{~d},{ }^{3} J_{\mathrm{H}, \mathrm{H}}=6.09 \mathrm{~Hz}, 12 \mathrm{H}, \mathrm{OCH} M e\right), 2.09$ $(\mathrm{s}, 6 \mathrm{H},=\mathrm{CMe}), 4.87\left(\mathrm{~m},{ }^{3} \mathrm{~J}_{\mathrm{H}, \mathrm{H}}=6.13 \mathrm{~Hz}, 2 \mathrm{H}, \mathrm{OCH}\right), 5.02$ $\left(\mathrm{m},{ }^{3} J_{\mathrm{H}, \mathrm{H}}=5.90 \mathrm{~Hz}, 4 \mathrm{H}, \quad\right.$ OCH $), \quad 5.30-5.45(\mathrm{~m}, 6 \mathrm{H}$, $\left.\mathrm{OCH}+=\mathrm{CH}_{2}\right), \quad 5.74 \quad\left(\mathrm{ddd}, \quad{ }^{2} J_{\mathrm{H}, \mathrm{H}}=3.50 \mathrm{~Hz}\right.$, $\left.{ }^{3} J_{\mathrm{H}, \mathrm{H}}=12.03 \mathrm{~Hz}, \quad{ }^{3} J_{\mathrm{P}, \mathrm{H}}=49.42 \mathrm{~Hz}, \quad 4 \mathrm{H}, \quad \mathrm{OCH}\right)$, 6.21-6.57 $\left(\mathrm{m}, \quad 6 \mathrm{H}, \quad \mathrm{CH}_{2}\right.$ (vinyl) $+\mathrm{CH}($ vinyl $)+=\mathrm{CH}_{2}$ (OMc) $)$ ppm; ${ }^{31} \mathrm{P} \quad \mathrm{NMR} \quad\left(\mathrm{C}_{6} \mathrm{D}_{6}, \quad 101.2 \mathrm{MHz}\right): \delta=$ $14.25 \mathrm{ppm} ;{ }^{13} \mathrm{C}$ NMR $\left(\mathrm{C}_{6} \mathrm{D}_{6}, 62.9 \mathrm{MHz}\right): \delta=18.58$ $(=\mathrm{CMe}), \quad 23.93 \quad$ (OCHMe $), 24.23 \quad$ (OCHMe), 24.78 (OCHMe), 25.17 (OCHMe), $77.82(\mathrm{OCH}), 78.65(\mathrm{OCH})$, $79.38 \quad(\mathrm{OCH}), 123.16 \quad\left(=\mathrm{CH}_{2} \quad(\mathrm{OMc}), 128.66 \quad\left(=\mathrm{CH}_{2}\right.\right.$ (vinyl)), $130.81\left(\mathrm{~d}\left({ }^{1} J_{\mathrm{P}, \mathrm{C}}=204.2 \mathrm{~Hz}\right), \mathrm{CH}\right.$ (vinyl) $), 140.22$ (=CMe), $173.41(\mathrm{COO}) \mathrm{ppm}$.

7: yield $160 \mathrm{mg}(34 \%) ;{ }^{1} \mathrm{H}$ NMR $\left(\mathrm{C}_{6} \mathrm{D}_{6}, 250 \mathrm{MHz}\right)$ : $\delta=1.30\left(\mathrm{~d},{ }^{3} J_{\mathrm{H}, \mathrm{H}}=6.09 \mathrm{~Hz}, 12 \mathrm{H}\right.$, OCHMe $), 1.41-1.49$ $(\mathrm{m}, 24 \mathrm{H}, \quad$ OCHMe $), 1.65\left(\mathrm{~d},{ }^{3} J_{\mathrm{H}, \mathrm{H}}=6.24 \mathrm{~Hz}, 12 \mathrm{H}\right.$, $\mathrm{OCHMe}), 1.72\left(\mathrm{~d},{ }^{3} J_{\mathrm{H}, \mathrm{H}}=6.24 \mathrm{~Hz}, 12 \mathrm{H}, \mathrm{OCHMe}\right), 2.07$ $(\mathrm{s}, 6 \mathrm{H},=\mathrm{CMe}), 3.20\left(\mathrm{~d},{ }^{2} J_{\mathrm{P}, \mathrm{H}}=22.69 \mathrm{~Hz}, 4 \mathrm{H}, \mathrm{PCH}_{2}\right)$, $4.83-5.02\left(\mathrm{~m},{ }^{3} J_{\mathrm{H}, \mathrm{H}}=6.07 \mathrm{~Hz}, 6 \mathrm{H}, \mathrm{OCH}\right), 5.18-5.33(\mathrm{~m}$, $\left.{ }^{3} J_{\mathrm{H}, \mathrm{H}}=6.24 \mathrm{~Hz}, 6 \mathrm{H}, \mathrm{OCH}\right), 5.40\left(\mathrm{br}, 2 \mathrm{H},=\mathrm{CH}_{2}\right), 6.32$ (br, $\left.2 \mathrm{H},=\mathrm{CH}_{2}\right), 7.18-7.28(\mathrm{~m}, 2 \mathrm{H}, \mathrm{Ph}), 7.31-7.39(\mathrm{~m}, 4 \mathrm{H}$, $\mathrm{Ph}),{ }^{7.63-7.68}(\mathrm{~m}, 4 \mathrm{H}, \mathrm{Ph}) \mathrm{ppm} ;{ }^{31} \mathrm{P}$ NMR $\left(\mathrm{C}_{6} \mathrm{D}_{6}\right.$, $101.2 \mathrm{MHz}): \quad \delta=23.34 \mathrm{ppm} ;{ }^{13} \mathrm{C} \quad \mathrm{NMR} \quad\left(\mathrm{C}_{6} \mathrm{D}_{6}\right.$, $62.9 \mathrm{MHz}): \delta=18.64$ (=CMe), 23.81 (OCHMe), 24.15 (OCHMe), 24.95 (OCHMe), 25.26 (OCHMe), 35.18 (d, 
$\left.{ }^{1} J_{\mathrm{P}, \mathrm{C}}=152.25 \mathrm{~Hz}, \mathrm{PCH}_{2}\right), 77.74(\mathrm{OCH}), 78.69(\mathrm{OCH})$, $79.05(\mathrm{OCH}), \quad 123.12 \quad\left(\mathrm{CH}_{2} \quad(\mathrm{OMc}), 125.83 \quad(\mathrm{~d}\right.$, $\left.J_{\mathrm{P}, \mathrm{C}}=3.00 \mathrm{~Hz}, \mathrm{Ph}\right), 130.44\left(\mathrm{~d}, J_{\mathrm{P}, \mathrm{C}}=6.98 \mathrm{~Hz}, \mathrm{Ph}\right)$, $134.88\left(\mathrm{~d}, J_{\mathrm{P}, \mathrm{C}}=8.98 \mathrm{~Hz}, \mathrm{Ph}\right), 140.19(=\mathrm{CMe}-), 173.34$ (COO) ppm.

8: yield $180 \mathrm{mg}(33 \%) ;{ }^{1} \mathrm{H}$ NMR $\left(\mathrm{C}_{6} \mathrm{D}_{6}, 250 \mathrm{MHz}\right)$ : $\delta=1.31\left(\mathrm{~d},{ }^{3} J_{\mathrm{H}, \mathrm{H}}=6.10 \mathrm{~Hz}, 12 \mathrm{H}, \mathrm{OCHMe}\right), 1.41(\mathrm{~d}$, $\left.{ }^{3} J_{\mathrm{H}, \mathrm{H}}=6.10 \mathrm{~Hz}, 12 \mathrm{H}, \mathrm{OCHMe}\right), 1.50\left(\mathrm{~d},{ }^{3} J_{\mathrm{H}, \mathrm{H}}=6.03 \mathrm{~Hz}\right.$, $12 \mathrm{H}, \mathrm{OCHMe}), 1.77\left(\mathrm{~d},{ }^{3} J_{\mathrm{H}, \mathrm{H}}=6.24 \mathrm{~Hz}, 12 \mathrm{H}, \mathrm{OCH} M e\right)$, $1.84\left(\mathrm{~d},{ }^{3} J_{\mathrm{H}, \mathrm{H}}=6.21 \mathrm{~Hz}, 12 \mathrm{H}\right.$, OCHMe $), 2.09(\mathrm{~s}, 6 \mathrm{H}$, $=\mathrm{CMe}), 2.68\left(\mathrm{dd},{ }^{2} J_{\mathrm{P}, \mathrm{H}}=22.69 \mathrm{~Hz},{ }^{3} J_{\mathrm{H}, \mathrm{H}}=7.08 \mathrm{~Hz}, 4 \mathrm{H}\right.$, $\left.\mathrm{PCH}_{2}\right), 4.85\left(\mathrm{~m},{ }^{3} J_{\mathrm{H}, \mathrm{H}}=6.09 \mathrm{~Hz}, 2 \mathrm{H}, \mathrm{OCH}\right), 5.00(\mathrm{~m}$, $\left.{ }^{3} J_{\mathrm{H}, \mathrm{H}}=6.05 \mathrm{~Hz}, \quad 4 \mathrm{H}, \quad \mathrm{OCH}\right), \quad 5.22-5.45 \quad(\mathrm{~m}, \quad 10 \mathrm{H}$, $\mathrm{OCH}+\mathrm{CH}_{2}(\mathrm{OMc})+\mathrm{CH}_{2}($ allyl $\left.)\right), 6.16-6.35(\mathrm{~m}, 2 \mathrm{H}, \mathrm{CH}$ (allyl)), 6.38 (br, 2H, $\left.=\mathrm{CH}_{2}(\mathrm{OMc})\right) \mathrm{ppm} ;{ }^{31} \mathrm{P} \mathrm{NMR}$ $\left(\mathrm{C}_{6} \mathrm{D}_{6}, 101.2 \mathrm{MHz}\right): \delta=24.16 \mathrm{ppm} ;{ }^{13} \mathrm{C} \mathrm{NMR}\left(\mathrm{C}_{6} \mathrm{D}_{6}\right.$, $62.9 \mathrm{MHz}): \delta=18.59$ (=CMe), 23.95 (OCHMe), 24.21 (OCHMe), 24.75 (OCHMe), 25.17 (OCHMe), 33.31 (d, $\left.{ }^{1} J_{\mathrm{P}, \mathrm{C}}=154.33 \mathrm{~Hz}, \mathrm{PCH}_{2}\right), 77.70(\mathrm{OCH}), 78.59(\mathrm{OCH})$, $79.31(\mathrm{OCH}), \quad 117.29\left(\mathrm{~d},{ }^{3} J_{\mathrm{P}, \mathrm{C}}=14.96 \mathrm{~Hz},=\mathrm{CH}_{2}\right)$, $123.14\left(\mathrm{CH}_{2}(\mathrm{OMc})\right), 130.96\left(\mathrm{~d},{ }^{2} J_{\mathrm{P}, \mathrm{C}}=10.97 \mathrm{~Hz},=\mathrm{CH}\right)$, 140.20 (C (OMc)), 173.28 (COO) ppm.

9: yield $100 \mathrm{mg}(22 \%) ;{ }^{1} \mathrm{H} \mathrm{NMR}\left(\mathrm{C}_{6} \mathrm{D}_{6}, 250 \mathrm{MHz}\right)$ : $\delta=1.31\left(\mathrm{~d},{ }^{3} J_{\mathrm{H}, \mathrm{H}}=6.10 \mathrm{~Hz}, 12 \mathrm{H}\right.$, OCHMe $), 1.42(\mathrm{~d}$, $\left.{ }^{3} J_{\mathrm{H}, \mathrm{H}}=6.15 \mathrm{~Hz}, 12 \mathrm{H}, \mathrm{OCHMe}\right), 1.50\left(\mathrm{~d},{ }^{3} J_{\mathrm{H}, \mathrm{H}}=6.08 \mathrm{~Hz}\right.$, $12 \mathrm{H}, \mathrm{OCHMe}), 1.76\left(\mathrm{~d},{ }^{3} J_{\mathrm{H}, \mathrm{H}}=6.24 \mathrm{~Hz}, 12 \mathrm{H}, \mathrm{OCH} M e\right)$, $1.82\left(\mathrm{~d},{ }^{3} J_{\mathrm{H}, \mathrm{H}}=6.20 \mathrm{~Hz}, 12 \mathrm{H}, \mathrm{OCHMe}\right), 1.89\left(\mathrm{~s}, 6 \mathrm{H}, \mathrm{CH}_{3}\right.$ (OMc ester)), $2.10(\mathrm{~s}, 6 \mathrm{H},=\mathrm{CMe}), 2.33-2.50(\mathrm{~m}, 4 \mathrm{H}$, $\left.\mathrm{PCH}_{2}\right), 4.79-5.06\left(\mathrm{~m}, 10 \mathrm{H}, \mathrm{OCH}+\mathrm{CH}_{2} \mathrm{O}\right), 5.26(\mathrm{br}, 2 \mathrm{H}$, $\mathrm{CH}_{2}$ (OMc ester)), $5.36\left(\mathrm{~m},{ }^{3} J_{\mathrm{H}, \mathrm{H}}=6.24 \mathrm{~Hz}, 4 \mathrm{H}, \mathrm{OCH}\right)$, 5.43 (br, $\left.2 \mathrm{H},=\mathrm{CH}_{2}\right), 6.20$ (br, $2 \mathrm{H}, \mathrm{CH}_{2}$ (OMc ester)), 6.38 (br, $\left.2 \mathrm{H},=\mathrm{CH}_{2}\right)$ ppm; ${ }^{31} \mathrm{P} \mathrm{NMR}\left(\mathrm{C}_{6} \mathrm{D}_{6}, 101.2 \mathrm{MHz}\right)$ : $\delta=23.59 \mathrm{ppm} ;{ }^{13} \mathrm{C} \mathrm{NMR}\left(\mathrm{C}_{6} \mathrm{D}_{6}, 62.9 \mathrm{MHz}\right): \delta=18.03$ $\left(\mathrm{CH}_{3}\right.$ (OMc ester)), 18.60 (=CMe), 23.90 (OCHMe), 24.19 (OCHMe), 24.71 (OCHMe), 25.16 (OCHMe), 27.88 (d, $\left.{ }^{1} J_{\mathrm{P}, \mathrm{C}}=152.46 \mathrm{~Hz}, \mathrm{PCH}_{2}\right), 60.92\left(\mathrm{~d},{ }^{2} J_{\mathrm{P}, \mathrm{C}}=3.98 \mathrm{~Hz}\right.$, $\left.\mathrm{OCH}_{2}\right), 78.03(\mathrm{OCH}), 78.80(\mathrm{OCH}), 79.71(\mathrm{OCH}), 123.67$ $\left(\mathrm{CH}_{2}(\mathrm{OMc}), 124.64\left(\mathrm{CH}_{2}\right.\right.$ (OMc ester)), 136.68 (C (OMc ester)), 139.94 (=CMe-), 166.56 (COO (OMc ester)), 173.50 (COO) ppm.

10: yield $120 \mathrm{mg}(24 \%) ;{ }^{1} \mathrm{H} \mathrm{NMR}\left(\mathrm{C}_{6} \mathrm{D}_{6}, 250 \mathrm{MHz}\right)$ : $\delta=1.26\left(\mathrm{~d},{ }^{3} J_{\mathrm{H}, \mathrm{H}}=6.09 \mathrm{~Hz}, 12 \mathrm{H}\right.$, OCHMe $), 1.38(\mathrm{~d}$, $\left.{ }^{3} J_{\mathrm{H}, \mathrm{H}}=6.24 \mathrm{~Hz}, 12 \mathrm{H}, \mathrm{OCHMe}\right), 1.41\left(\mathrm{~d},{ }^{3} J_{\mathrm{H}, \mathrm{H}}=6.09 \mathrm{~Hz}\right.$, $12 \mathrm{H}, \mathrm{OCHMe}), 1.67\left(\mathrm{~d},{ }^{3} J_{\mathrm{H}, \mathrm{H}}=6.24 \mathrm{~Hz}, 12 \mathrm{H}, \mathrm{OCH} M e\right)$, $1.77\left(\mathrm{~d},{ }^{3} J_{\mathrm{H}, \mathrm{H}}=6.24 \mathrm{~Hz}, 12 \mathrm{H}\right.$, OCHMe $), 1.80-1.89(\mathrm{~m}$, $\left.4 \mathrm{H}, \mathrm{CH}_{2} \mathrm{CN}\right), 2.02(\mathrm{~s}, 6 \mathrm{H},=\mathrm{CMe}), 2.54-2.66(\mathrm{~m}, 4 \mathrm{H}$, $\left.\mathrm{PCH}_{2}\right), 4.79$ (m, 2H, OCH), 4.89 (m, 4H, OCH), 5.29 (m, $4 \mathrm{H}, \mathrm{OCH}), 5.36\left(\mathrm{br}, 2 \mathrm{H},=\mathrm{CH}_{2}\right), 6.29\left(\mathrm{br}, 2 \mathrm{H},=\mathrm{CH}_{2}\right) \mathrm{ppm}$; ${ }^{31} \mathrm{P}$ NMR $\left(\mathrm{C}_{6} \mathrm{D}_{6}, 101.2 \mathrm{MHz}\right): \delta=24.16 \mathrm{ppm} ;{ }^{13} \mathrm{C} \mathrm{NMR}$ $\left(\mathrm{C}_{6} \mathrm{D}_{6}, \quad 62.9 \mathrm{MHz}\right): \quad \delta=11.67 \quad\left(\mathrm{~d}, \quad{ }^{2} J_{\mathrm{P}, \mathrm{C}}=2.50 \mathrm{~Hz}\right.$, $\left.\mathrm{CH}_{2} \mathrm{CN}\right), 18.46$ (=CMe), $22.04\left(\mathrm{PCH}_{2}\right), 23.80(\mathrm{OCH} M e)$, 24.16 (OCHMe), 24.63 (OCHMe), 25.05 (OCHMe), 25.11 $(\mathrm{OCHMe}), 78.13(\mathrm{OCH}), 78.94(\mathrm{OCH}), 79.96(\mathrm{OCH})$, $118.86\left(\mathrm{~d},{ }^{3} J_{\mathrm{P}, \mathrm{C}}=18.95 \mathrm{~Hz}, \mathrm{CN}\right), 123.61\left(=\mathrm{CH}_{2}\right), 139.83$ (=CMe-), 173.48 (COO) ppm.

11: yield $230 \mathrm{mg}(48 \%) ;{ }^{1} \mathrm{H}$ NMR $\left(\mathrm{C}_{6} \mathrm{D}_{6}, 250 \mathrm{MHz}\right)$ : $\delta=1.23-1.47 \quad\left(\mathrm{~m}, \quad 6 \mathrm{H}, \quad \mathrm{CH}_{3} \mathrm{CH}_{2} \mathrm{P}\right), \quad 1.32 \quad(\mathrm{~d}$, $\left.{ }^{3} J_{\mathrm{H}, \mathrm{H}}=6.09 \mathrm{~Hz}, 12 \mathrm{H}, \mathrm{OCHMe}\right), 1.41\left(\mathrm{~d},{ }^{3} J_{\mathrm{H}, \mathrm{H}}=6.24 \mathrm{~Hz}\right.$, $12 \mathrm{H}, \mathrm{OCHMe}), 1.50\left(\mathrm{~d},{ }^{3} J_{\mathrm{H}, \mathrm{H}}=6.09 \mathrm{~Hz}, 12 \mathrm{H}, \mathrm{OCHMe}\right)$, 1.65-1.93 (m, 4H, PCH 2$), 1.77\left(\mathrm{~d},{ }^{3} J_{\mathrm{H}, \mathrm{H}}=6.24 \mathrm{~Hz}, 12 \mathrm{H}\right.$, $\mathrm{OCHMe}), 1.84\left(\mathrm{~d},{ }^{3} J_{\mathrm{H}, \mathrm{H}}=6.24 \mathrm{~Hz}, 12 \mathrm{H}, \mathrm{OCH} M e\right), 2.09$ $(\mathrm{s}, 6 \mathrm{H},=\mathrm{CMe}), 4.86\left(\mathrm{~m},{ }^{3} J_{\mathrm{H}, \mathrm{H}}=6.09 \mathrm{~Hz}, 2 \mathrm{H}, \mathrm{OCH}\right), 5.00$ $\left(\mathrm{m},{ }^{3} J_{\mathrm{H}, \mathrm{H}}=6.09 \mathrm{~Hz}, 4 \mathrm{H}, \mathrm{OCH}\right), 5.27-5.37(\mathrm{~m}, 4 \mathrm{H}, \mathrm{OCH})$, 5.39 (br, 2H, $\left.\mathrm{CH}_{2}(\mathrm{OMc})\right), 6.36$ (br, 2H, $\left.\mathrm{CH}_{2}(\mathrm{OMc})\right)$ ppm; ${ }^{31} \mathrm{P}$ NMR $\left(\mathrm{C}_{6} \mathrm{D}_{6}, 101.2 \mathrm{MHz}\right): \delta=29.82 \mathrm{ppm} ;{ }^{13} \mathrm{C} \mathrm{NMR}$ $\left(\mathrm{C}_{6} \mathrm{D}_{6}, \quad 62.9 \mathrm{MHz}\right): \quad \delta=7.42 \quad\left(\mathrm{~d}, \quad{ }^{2} J_{\mathrm{P}, \mathrm{C}}=6.28 \mathrm{~Hz}\right.$, $\left.\mathrm{CH}_{3} \mathrm{CH}_{2} \mathrm{P}\right), 18.58$ (=CMe), $20.00\left(\mathrm{~d},{ }^{1} J_{\mathrm{P}, \mathrm{C}}=158.57 \mathrm{~Hz}\right.$, $\mathrm{PCH}_{2}$ ), 23.93 (OCHMe), 24.20 (OCHMe), 24.75 (OCHMe), 25.13 (OCHMe), 25.21 (OCHMe), 77.51 (OCH), 78.44 $(\mathrm{OCH}), 79.09(\mathrm{OCH}), 122.99\left(=\mathrm{CH}_{2}\right), 140.28(=\mathrm{CMe}-)$, 173.23 (COO) ppm.

12: yield $260 \mathrm{mg}(53 \%) ;{ }^{1} \mathrm{H}$ NMR $\left(\mathrm{C}_{6} \mathrm{D}_{6}, 250 \mathrm{MHz}\right)$ : $\delta=1.32\left(\mathrm{~d},{ }^{3} J_{\mathrm{H}, \mathrm{H}}=6.09 \mathrm{~Hz}, 12 \mathrm{H}, \mathrm{OCHMe}\right), 1.39(\mathrm{~d}$, $\left.{ }^{3} J_{\mathrm{H}, \mathrm{H}}=6.24 \mathrm{~Hz}, 12 \mathrm{H}, \mathrm{OCHMe}\right), 1.48\left(\mathrm{~d},{ }^{3} J_{\mathrm{H}, \mathrm{H}}=6.09 \mathrm{~Hz}\right.$, $12 \mathrm{H}, \mathrm{OCHMe}), 1.71\left(\mathrm{~d},{ }^{3} J_{\mathrm{H}, \mathrm{H}}=6.24 \mathrm{~Hz}, 12 \mathrm{H}, \mathrm{OCHMe}\right)$, $1.80\left(\mathrm{~d},{ }^{3} J_{\mathrm{H}, \mathrm{H}}=6.24 \mathrm{~Hz}, 12 \mathrm{H}, \mathrm{OCHMe}\right), 2.05(\mathrm{~s}, 6 \mathrm{H}$, $=\mathrm{CMe}), 2.46(\mathrm{~m}, 4 \mathrm{H}, \mathrm{MeCO}), 3.00\left(\mathrm{~d},{ }^{2} J_{\mathrm{P}, \mathrm{H}}=23.91 \mathrm{~Hz}\right.$, $\left.4 \mathrm{H}, \mathrm{PCH}_{2}\right), 4.81\left(\mathrm{~m},{ }^{3} J_{\mathrm{H}, \mathrm{H}}=6.17 \mathrm{~Hz}, 2 \mathrm{H}, \mathrm{OCH}\right), 4.98(\mathrm{~m}$, $\left.{ }^{3} J_{\mathrm{H}, \mathrm{H}}=6.13 \mathrm{~Hz}, 4 \mathrm{H}, \mathrm{OCH}\right), 5.31\left(\mathrm{~m},{ }^{3} J_{\mathrm{H}, \mathrm{H}}=6.32 \mathrm{~Hz}\right.$, $4 \mathrm{H}, \mathrm{OCH}), 5.38$ (br, 2H, $\left.\mathrm{CH}_{2}(\mathrm{OMc})\right), 6.32\left(\mathrm{br}, 2 \mathrm{H},=\mathrm{CH}_{2}\right)$ ppm; ${ }^{31} \mathrm{P}$ NMR $\left(\mathrm{C}_{6} \mathrm{D}_{6}, 101.2 \mathrm{MHz}\right): \delta=18.69 \mathrm{ppm} ;{ }^{13} \mathrm{C}$ NMR $\left(\mathrm{C}_{6} \mathrm{D}_{6}, \quad 62.9 \mathrm{MHz}\right): \delta=18.51 \quad$ (=CMe), 23.80 $(\mathrm{OCHMe}), 24.19$ (OCHMe), 24.72 (OCHMe), 25.20 (OCHMe $), 30.50\left(\mathrm{CH}_{3}-\mathrm{CO}\right), 45.25\left(\mathrm{~d},{ }^{1} J_{\mathrm{P}, \mathrm{C}}=139.17 \mathrm{~Hz}\right.$, $\left.\mathrm{PCH}_{2}\right), 78.29(\mathrm{OCH}), 78.94(\mathrm{OCH}), 79.90(\mathrm{OCH}), 123.59$ $\left(\mathrm{CH}_{2}(\mathrm{OMc})\right), 139.89$ (C (OMc)), 173.48 (COO), 199.11 (d, $\left.{ }^{2} J_{\mathrm{P}, \mathrm{C}}=5.58 \mathrm{~Hz}, \mathrm{C}=\mathrm{O}\right) \mathrm{ppm}$.

\section{$X$-ray structure analyses}

All measurements were performed using $\mathrm{Mo} K_{\alpha}$ radiation $(\lambda=71.073 \mathrm{pm})$. Data were collected on a Bruker Axs SMART APEX II four-circle diffractometer with $\kappa$-geometry at $100 \mathrm{~K}$ with $\varphi$ and $\omega$-scans and $0.5^{\circ}$ frame width (Table 1) and corrected for polarization and Lorentz effects. An empirical absorption correction (SADABS) was applied. The cell dimensions were refined with all unique reflections. Saint Plus (Bruker Analytical X-ray Instruments, 2007) was used to integrate the frames. Symmetry was checked with the program Platon.

The structures were solved by the Patterson method (SHELXS97). Refinement was performed by the full-matrix least-squares method based on $F^{2}$ (SHELXL97) with anisotropic thermal parameters for all non-hydrogen atoms. Hydrogen atoms were inserted in calculated positions and refined riding with the corresponding atom. In 1, 3, 4, 6-9, 
Table 1 Crystal data and structure refinement details

\begin{tabular}{|c|c|c|c|c|}
\hline Compound & 1 & 2 & 3 & 4 \\
\hline Emp. formula & $\mathrm{C}_{43} \mathrm{H}_{97} \mathrm{O}_{23} \mathrm{P}_{3} \mathrm{Ti}_{5}$ & $\mathrm{C}_{46} \mathrm{H}_{100} \mathrm{Br}_{3} \mathrm{O}_{23} \mathrm{P}_{3} \mathrm{Ti}_{5}$ & $\mathrm{C}_{94} \mathrm{H}_{182} \mathrm{O}_{54} \mathrm{P}_{10} \mathrm{Ti}_{10}$ & $\mathrm{C}_{44} \mathrm{H}_{92} \mathrm{Br}_{2} \mathrm{O}_{24} \mathrm{P}_{2} \mathrm{Ti}_{6}$ \\
\hline$M_{r}$ & 1314.62 & 1593.4 & 2965.1 & 1514.35 \\
\hline Crystal system & Monoclinic & Monoclinic & Triclinic & Monoclinic \\
\hline Space group & $P 2_{1} / c$ & $P c$ & $P \overline{1}$ & $P 2_{1} / n$ \\
\hline a/pm & $2756.6(1)$ & $1291.63(5)$ & $1366.83(13)$ & $1346.04(2)$ \\
\hline$b / \mathrm{pm}$ & $1861.8(1)$ & $2301.65(9)$ & $1403.15(13)$ & $1532.03(3)$ \\
\hline$c / \mathrm{pm}$ & $2600.8(1)$ & $2441.36(9)$ & 2110.25 (17) & $1689.82(3)$ \\
\hline$\alpha /^{\circ}$ & 90 & 90 & $73.525(4)$ & 90 \\
\hline$\beta /^{\circ}$ & $105.268(2)$ & $101.4782(14)$ & 75.199 (4) & $108.9870(10)$ \\
\hline$\gamma /{ }^{\circ}$ & 90 & 90 & $64.922(5)$ & 90 \\
\hline$V / \mathrm{pm}^{3} \times 10^{6}$ & $12877(1)$ & $7112.7(5)$ & $3472.3(5)$ & $3295.11(10)$ \\
\hline$Z$ & 8 & 4 & 1 & 2 \\
\hline$D_{x} / \mathrm{g} \mathrm{cm}^{-3}$ & 1.36 & 1.488 & 1.418 & 1.526 \\
\hline$\mu / \mathrm{mm}^{-1}$ & 0.735 & 2.355 & 0.739 & 2.023 \\
\hline Crystal size/mm & $0.25 \times 0.2 \times 0.15$ & $0.52 \times 0.15 \times 0.1$ & $0.25 \times 0.15 \times 0.1$ & $0.48 \times 0.44 \times 0.4$ \\
\hline No. measured refl. & 230,762 & 101,719 & 76,823 & 43500 \\
\hline Obs. refl. $[I>2 \sigma(I)]$ & 17,441 & 23,566 & 8533 & 8180 \\
\hline$\theta_{\max } /{ }^{\circ}$ & 25.07 & 26.37 & 25.15 & 30.55 \\
\hline $\mathrm{R}\left[F^{2}>2 \sigma(F)\right], \mathrm{w} R\left(F^{2}\right), S$ & $0.0355,0.0965,1.074$ & $0.0433,0.1134,1.076$ & $0.0429,0.1083,1.035$ & $0.0386,0.1101,1.089$ \\
\hline Refl./param. & $22815 / 1413$ & $27296 / 1497$ & $12361 / 828$ & $10086 / 393$ \\
\hline Weighting scheme ${ }^{a}$ & $a=0.0409 \mathrm{P}, b=14.5476$ & $a=0.0588, b=8.0538$ & $a=0.0457, b=3.3285$ & $a=0.0621, b=0.1815$ \\
\hline$\delta \rho_{\max }, \min _{1} / \mathrm{e} \times 10^{-6} \mathrm{pm}^{-3}$ & $1.00,-0.92$ & $1.418,-1.716$ & $0.78,-0.49$ & $1.329,-1.132$ \\
\hline Compound & 5 & 6 & 7 & 8 \\
\hline Emp. formula & $\mathrm{C}_{50} \mathrm{H}_{90} \mathrm{O}_{24} \mathrm{P}_{2} \mathrm{Ti}_{6}$ & $\mathrm{C}_{42} \mathrm{H}_{84} \mathrm{O}_{24} \mathrm{P}_{2} \mathrm{Ti}_{6}$ & $\mathrm{C}_{52} \mathrm{H}_{94} \mathrm{O}_{24} \mathrm{P}_{2} \mathrm{Ti}_{6}$ & $\mathrm{C}_{44} \mathrm{H}_{90} \mathrm{O}_{24} \mathrm{P}_{2} \mathrm{Ti}_{6}$ \\
\hline$M_{\mathrm{r}}$ & 1424.56 & 1322.43 & 1452.61 & 1352.5 \\
\hline Crystal system & Monoclinic & Triclinic & Monoclinic & Triclinic \\
\hline Space group & $P 2_{1} / n$ & $P \overline{1}$ & $P 2_{1} / n$ & $P \overline{1}$ \\
\hline$a / \mathrm{pm}$ & $1388.4(1)$ & $1162.8(1)$ & $1376.21(9)$ & $1270.28(9)$ \\
\hline$b / \mathrm{pm}$ & $1742.4(2)$ & $1264.4(2)$ & $1326.97(8)$ & $1443.4(1)$ \\
\hline$c / \mathrm{pm}$ & $1411.0(1)$ & $1265.7(2)$ & $1936.70(11)$ & $1838.2(1)$ \\
\hline$\alpha /^{\circ}$ & 90 & $107.721(3)$ & 90 & $92.141(3)$ \\
\hline$\beta /^{\circ}$ & $91.423(3)$ & $95.875(3)$ & $101.120(2)$ & $90.567(3)$ \\
\hline$\gamma /{ }^{\circ}$ & 90 & $113.308(3)$ & 90 & $94.006(3)$ \\
\hline$V / \mathrm{pm}^{3} \times 10^{6}$ & $3412.4(5)$ & $1574.4(3)$ & $3470.4(4)$ & $3359.7(4)$ \\
\hline$Z$ & 2 & 1 & 2 & 2 \\
\hline$D_{x} / \mathrm{g} \mathrm{cm}^{-3}$ & 1.386 & 1.395 & 1.39 & 1.337 \\
\hline$\mu / \mathrm{mm}^{-1}$ & 0.785 & 0.844 & 0.773 & 0.793 \\
\hline Crystal size/mm & $0.45 \times 0.42 \times 0.38$ & $0.42 \times 0.38 \times 0.37$ & $0.38 \times 0.37 \times 0.3$ & $0.42 \times 0.37 \times 0.34$ \\
\hline No. measured refl. & 86,933 & 37,906 & 42,971 & 81,110 \\
\hline Obs. refl. $[I>2 \sigma(I)]$ & 5075 & 3906 & 9040 & 12,077 \\
\hline$\theta_{\max } /{ }^{\circ}$ & 26.4 & 25.14 & 30.51 & 28.6 \\
\hline $\mathrm{R}\left[F^{2}>2 \sigma(F)\right], \mathrm{w} R\left(F^{2}\right), S$ & $0.0869,0.2767,1.171$ & $0.0928,0.2097,1.059$ & $0.0322,0.1005,1.088$ & $0.0588,0.1819,1.075$ \\
\hline Refl./param. & $6991 / 382$ & $5595 / 413$ & $10,593 / 495$ & $16,783 / 799$ \\
\hline Weighting scheme $^{\mathrm{a}}$ & $a=0.1198, b=22.3579$ & $a=0.0146, b=20.0056$ & $a=0.0503, b=2.2610$ & $a=0.0765, b=4.8222$ \\
\hline$\delta \rho_{\max }, \min / \mathrm{e} \times 10^{-6} \mathrm{pm}^{-3}$ & $1.945,-0.978$ & $1.541,-0.836$ & $1.131,-0.82$ & $1.317,-0.753$ \\
\hline
\end{tabular}


Table 1 continued

\begin{tabular}{|c|c|c|c|c|}
\hline Compound & 9 & 10 & 11 & 12 \\
\hline Emp. formula & $\mathrm{C}_{50} \mathrm{H}_{98} \mathrm{O}_{28} \mathrm{P}_{2} \mathrm{Ti}_{6}$ & $\mathrm{C}_{44} \mathrm{H}_{87} \mathrm{~N}_{2} \mathrm{O}_{24} \mathrm{P}_{2} \mathrm{Ti}_{6}$ & $\mathrm{C}_{42} \mathrm{H}_{90} \mathrm{O}_{24} \mathrm{P}_{2} \mathrm{Ti}_{6}$ & $\mathrm{C}_{44} \mathrm{H}_{90} \mathrm{O}_{26} \mathrm{P}_{2} \mathrm{Ti}_{6}$ \\
\hline$M_{\mathrm{r}}$ & 1496.62 & 1377.5 & 1328.48 & 1384.5 \\
\hline Crystal system & Triclinic & Monoclinic & Triclinic & Monoclinic \\
\hline Space group & $P \overline{1}$ & $P 2_{1} / c$ & $P \overline{1}$ & $C 2 / c$ \\
\hline$a / \mathrm{pm}$ & $1316.93(9)$ & $1993.57(3)$ & $1191.1(1)$ & $2114.2(6)$ \\
\hline$b / \mathrm{pm}$ & $1318.99(9)$ & $1909.15(3)$ & $1254.1(1)$ & $1306.8(3)$ \\
\hline$c / \mathrm{pm}$ & $2275.93(16)$ & $1830.71(3)$ & $1321.9(1)$ & $2454.2(7)$ \\
\hline$\alpha /^{\circ}$ & $93.586(2)$ & 90 & $67.017(3)$ & 90 \\
\hline$\beta /^{\circ}$ & $98.919(2)$ & $99.4200(10)$ & $89.871(3)$ & $104.112(8)$ \\
\hline$\gamma /{ }^{\circ}$ & 113.5566 (19) & 90 & $65.009(3)$ & 90 \\
\hline$V / \mathrm{pm}^{3} \times 10^{6}$ & $3546.1(4)$ & 6873.77 (19) & $1616.0(3)$ & $6576(3)$ \\
\hline$Z$ & 2 & 4 & 1 & 4 \\
\hline$D_{x} / \mathrm{g} \mathrm{cm}^{-3}$ & 1.402 & 1.331 & 1.365 & 1.399 \\
\hline$\mu / \mathrm{mm}^{-1}$ & 0.763 & 0.777 & 0.823 & 0.814 \\
\hline Crystal size/mm & $0.45 \times 0.43 \times 0.4$ & $0.48 \times 0.42 \times 0.38$ & $0.55 \times 0.5 \times 0.45$ & $0.51 \times 0.41 \times 0.32$ \\
\hline No. measured refl. & 136,754 & 52,967 & 15,444 & 130,844 \\
\hline Obs. refl. $[I>2 \sigma(I)]$ & 17,747 & 8622 & 3838 & 7964 \\
\hline$\theta_{\max } /^{\circ}$ & 30.56 & 25.11 & 25.03 & 30.58 \\
\hline $\mathrm{R}\left[F^{2}>2 \sigma(F)\right], \mathrm{w} R\left(F^{2}\right), S$ & $0.0567,0.1295,1.079$ & $0.063,0.1999,1.074$ & $0.0922,0.2265,1.045$ & $0.067,0.1946,1.165$ \\
\hline Refl./param. & $21,716 / 894$ & $12,226 / 724$ & $5543 / 492$ & $10,080 / 407$ \\
\hline Weighting scheme ${ }^{a}$ & $a=0.0178, b=11.8092$ & $a=0.0994, b=8.5071$ & $a=0.0483, \mathrm{~b}=17.8591$ & $a=0.0659, \mathrm{~b}=45.3501$ \\
\hline$\delta \rho_{\max , \min } / \mathrm{e} \times 10^{-6} \mathrm{pm}^{-3}$ & $1.49,-1.873$ & $0.732,-0.342$ & $1.435,-0.834$ & $1.147,-0.653$ \\
\hline
\end{tabular}

${ }^{\mathrm{a}} w=\frac{1}{\sigma^{2}\left(F_{0}\right)^{2}+(a P)^{2}+b P}$ where $P=\frac{F_{0}^{2}+2 F_{\mathrm{c}}^{2}}{3}$

11, and 12 OiPr ligands were disordered. In 6 and 12 one OiPr ligand was additionally refined for three different positions. In 6, 9, and $\mathbf{1 1}$ the methacrylate ligand was bridging either between $\mathrm{Ti}(1)$ and $\mathrm{Ti}(2)$ or between $\mathrm{Ti}(1)$ and Ti(3). Two allyl groups in $\mathbf{3}$ and one $\mathrm{Br}$ atom in $\mathbf{2}$ were also disordered.

CCDC-1027711 (for 1), -1027712 (for 2), -1027713 (for 3), -1027714 (for 4), -1027715 (for 5), -1027716 (for 6), -1027717 (for 7), -1027718 (for 8), -1027719 (for 9), -1027720 (for 10), -1027721 (for 11), and -1027722 (for 12) contain the supplementary crystallographic data. These data can be obtained free of charge from the Cambridge Crystallographic Data Centre via http://www.ccdc.cam.ac. uk/data_request/cif.

Acknowledgments This work was supported by the Fonds zur Förderung der wissenschaftlichen Forschung (FWF), Austria (Project $\mathrm{P} 22915)$. The X-ray measurements were carried out at the X-ray Center of Vienna University of Technology.

Open Access This article is distributed under the terms of the Creative Commons Attribution 4.0 International License (http:// creativecommons.org/licenses/by/4.0/), which permits unrestricted use, distribution, and reproduction in any medium, provided you give appropriate credit to the original author(s) and the source, provide a link to the Creative Commons license, and indicate if changes were made.

\section{References}

1. Czakler M, Artner C, Schubert U (2014) Eur J Inorg Chem 2038

2. Schubert U (2001) Chem Mater 13:3487

3. Schubert U (2011) Chem Soc Rev 40:575

4. Guerrero G, Mehring M, Mutin PH, Dahan F, Vioux A (1999) J Chem Soc Dalton Trans 61:1537

5. Mehring M, Guerrero G, Dahan F, Mutin PH, Vioux A (2000) Inorg Chem 39:3325

6. Chakraborty D, Chandrasekhar V, Bhattacharjee M, Krätzner R, Roesky HW, Noltemeyer M, Schmidt H (2000) Inorg Chem 39:23

7. Czakler M, Artner C, Schubert U (2013) Eur J Inorg Chem 5790

8. Chandrasekhar V, Senapati T, Dey A, Hossain S (2011) Dalton Trans 40:5394

9. Czakler M, Artner C, Schubert U (2014) Monatsh Chem. doi:10. 1007/s00706-015-1443-6

10. Newman MS (1950) J Am Chem Soc 72:4783 